V. Alonso Sánchez Coello al cardenal Farnesio, Madrid, 28 de mayo de 1588. (A.S.Pr., Carteggio Farnesiano, Estero, Spagna, b.129).

$\mathrm{Ill}^{\mathrm{mo}} \mathrm{S}^{\text {or } ~ / ~ N o ~ p u e d o ~ d e x a r ~ d e ~ i m p o r t u n a r ~ a ~ v . s . I l l . ~ l a s ~ v e z e s ~ q u e ~ s e ~ o f f r e c i e r e ~ o c c a s i o n ~ y ~ t r a e r ~ a ~ l a ~ m e m o-~}$ ria al licenciado Juan Sánchez mi hijo, para que v.s.Ill ${ }^{\mathrm{ma}}$ La tenga de hazerle merced en alguna vacante, pues agora ay mejor ocasion de poderlo hazer por aver tomado su $\mathrm{s}^{\mathrm{d}}$ en si los indultos segun aca se dize que su s $\mathrm{s}^{\mathrm{d}}$ avia dado a los ss. cardenales: porque Le certifico a V. S. Ill. que en el tiempo que ha que reside en esa corte que son tres años, como esta a mi costa me ha gastado mucha hazienda, y me la gasta, que me ha puesto y pone en no pequeña necessidad, si v.s.Ill. no lo remedia como me lo tiene offrecido tantas vezes, yo no se que me haga sino es dar gracias a N.S. que ha sido servido y Lo deve ser desto, por lo cual supp ${ }^{\text {co }}$ a V. S.Ill, que pues Le tomo debaxo de su amparo y protección, y el por su persona no desmerece qualquiera mrd que se le haga: ni yo por mi voluntad y desseo de acertar a servir a V.s.Ill. tampoco, favorezca su causa de manera que salga cierta mi esperança y el con alguna cosa para que del todo yo no me pierda por su occasion, y pueda favorecer quatro hermanas donzellas que tiene, porque sentiría mucho que sabiendo aqui todos La mrd que V.s.Ill. haze a mi hijo, viessen despues que avia sido desfavorecido, pues pudiendolo V.s.Ill. todo el se quedava sin nada que segun son las gentes juzgarían que de meritos suyos devia ser la causa desto, yo quedo confiado que V.S.Ill, remediara estos daños con hazer de manera que se le de alguna vacante: si para alguna cosa del servi ${ }^{\circ}$ de v.s.Ill. fuere yo menester no tengo que offrecerme de nuevo quien ha tantos dias que Lo esta sino que V.S.Ill. me emplee en el pues sera para mi tanta mrd, N.S ${ }^{\mathrm{r}} \mathrm{g}^{\mathrm{e}}$ y acreciente La Ill $^{\mathrm{ma}}$ persona y gran estado

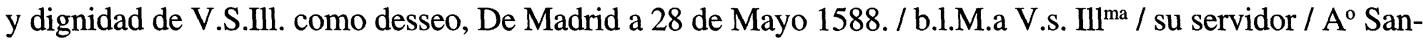
chez Coello.

\title{
UN RETRATO DE JUAN DE BRIVIESCA POR JERÓNIMO LÓPEZ POLANCO
}

Desde que el actual palacio episcopal de Segovia fuera adquirido en el siglo XVIII para servir de residencia a los obispos de la diócesis, el edificio de la plaza de San Esteban ha ido acumulando en su interior un variado, en cuanto a calidad y estilo, conjunto de objetos y obras de arte que han formado una valiosa y singular colección. La compra de obras con el fin de decorar las estancias del palacio, la desamortización eclesiástica del siglo XIX, el intento de crear un museo diocesano en 1921 o el despoblamiento del medio rural durante el siglo XX son algunos de los hechos que han contribuido a formar esta colección.

Aunque el palacio siempre tuvo un acceso restringido, muchas de sus mejores pinturas y esculturas han sido ya estudiadas y publicadas; otras, desconocidas, pero de indudable mérito, esperan aún el momento oportuno para salir a la luz. Gracias a la creación del Museo «Palacio Episcopal», inaugurado en junio de 1995, se ha conseguido no sólo la restauración y catalogación de una significativa parte de sus fondos, sino también la difusión y puesta en valor de los mismos.

El lienzo objeto de estas líneas es un retrato del sacerdote y terciario franciscano Juan de Briviesca, realizado por el pintor madrileño Jerónimo López Polanco, obra localizada en el palacio desde fecha desconocida y «rescatada» del lugar donde se encontraba almacenada para ser expuesta, tras su oportuna restauración, en una de las salas del museo (Figs. 11 y 12) ${ }^{1}$. En los es-

\footnotetext{
${ }^{1}$ La restauración, llevada a cabo en Junio de 1995, consistió en un forrado de la debilitada tela original, una sustitución del bastidor y un sentado y limpieza de la capa pictórica. Al carecer de marco, se adaptó uno de época que, sin lienzo, se conservaba en el Museo.
} 
cuetos inventarios de los siglos XIX y XX no se menciona este retrato, pero no es de extrañar, ya que en ellos sólo figuran los objetos que formaban parte de la decoración de las salas; esta obra, sin marco, con un pobre y deteriorado bastidor y en un delicado estado de conservación, no alcanzaba, pese a su calidad, el mínimo decoro entonces exigido para el ornamento del palacio.

Se trata de una pintura interesante no sólo por su evidente calidad pictórica y sugerente plasticidad, sino también por la atractiva personalidad del retratado y por lo desconocido del pintor; también la existencia en Segovia de otros dos lienzos con el mismo protagonista y semejantes características, procedentes de la colección del ceramista Daniel Zuloaga, aumenta el interés de la misma ${ }^{2}$.

El retrato, realizado hacia 1619, llama la atención desde un primer momento a quien se acerca a contemplarlo, pues en él está presente todo el sabor y el carácter de la mejor pintura española del primer tercio del siglo XVII. Jerónimo López Polanco se muestra en esta obra como uno de los pintores más capaces de la escuela madrileña del reinado de Felipe III; crea una obra basada en la verosimilitud, alejada de la idealización y volcada en un digno naturalismo, características presentes en las mejores realizaciones de esta escuela. Como ocurre con algunas obras de pintores madrileños de este período, el retrato de Juan de Briviesca presenta una gran cercanía con el estilo del joven Velázquez, pese a haber sido realizado antes del primer viaje a la Corte del genial sevillano.

La composición de esta pintura continúa la tradición de los retratos españoles de finales del siglo XVI y presenta al clérigo de medio cuerpo sobre fondo neutro sin referencia espacial alguna; la posición del personaje, ligeramente ladeado hacia su izquierda, favorece una completa visión del perfil derecho, perfil que también recibe una mayor iluminación respecto al contrario, donde domina una suave penumbra. Las oscuras vestiduras, apenas esbozadas, se confunden con el fondo tostado; de toda la indumentaria, camisa, sotana y capa, destaca el cuello de la primera, esmeradamente pintado en blanco, color que ayuda a resaltar el rostro; éste, rasurado a excepción del bigote, luce largas patillas y ordenado flequillo sobre la frente.

Es un retrato veraz del sacerdote que no pretende el halago a través de la idealización; el personaje, absorto en sus meditaciones, no dirige la mirada al espectador, sino que presenta una actitud introspectiva, sin relación con el exterior; la mirada fija, algo severa, y el gesto serio, cargado de determinación, nos dan una idea acerca del carácter que pudo tener nuestro ascético clérigo.

Ofrece Jerónimo López Polanco una técnica precisa, una gama de color extraordinariamente reducida y una factura pulida, poco empastada; la luz, algo tenebrista, modula el rostro y lo resalta del fondo. El hasta ahora poco conocido pintor madrileño, se nos muestra en esta obra como un excelente retratista que plasma en el lienzo con acierto no sólo los rasgos físicos del modelo, sino también el espíritu del mismo.

La cartela, escrita en 1716, coincidiendo con el desarrollo del proceso de beatificación del sacerdote, incide sobre la autenticidad y originalidad del retrato y nos revela la identidad del pintor y del personaje retratado, aportando también algunos datos biográficos de este último: «Verdadero, original. Retrato del Venerable Hermano licenciado Juan de Briviesca Pintado en los 34 años de su edad por Gerónimo López. Murió en los 41 años seis meses y ocho días de su edad en 5 de Marzo año de 1627 en Segovia en casa de D. Francisco de Riofrío Patrón de San Lorenzo. Yace en la Capilla de la Orden tercera de Nuestro Padre San Francisco en el arco que está del lado del evangelio. Trátase de su Beatificación. Mayo de 1716.»

La gran precisión en los datos escritos sobre el lienzo en 1716, teniendo en cuenta que ya

\footnotetext{
2 Ambas obras se encontraban hasta hace unos meses en un comercio de antigüedades de la Granja de San Ildefonso, aunque una de ellas, la de mayor calidad, ha pasado recientemente a una colección privada madrileña.
} 
habían pasado 89 años desde la muerte de Briviesca y 97 desde la realización de la pintura, parece indicar que el redactor del texto, muy posiblemente un terciario, pudo haber tenido en su poder la documentación necesaria en relación con el pintor y con el clérigo que le permitió conocer con certeza toda esa información; todos los datos ofrecidos en esta obra coinciden tanto con la vida de Juan de Briviesca, como con las fechas en que estuvo activo el autor del retrato.

Los otros dos lienzos arriba mencionados presentan una gran desigualdad en cuanto a su valor artístico; la pintura conservada en una colección privada madrileña es una obra muy cercana en cuanto a composición, estilo y calidad respecto a la del Museo, con la diferencia de no ofrecer dato alguno referente al personaje o al pintor; el lienzo existente en un comercio de antigüedades de San Ildefonso, pese a ser una copia de baja calidad, tiene un gran interés por la inscripción que presenta, ya que los datos allí ofrecidos han sido decisivos a la hora de buscar información sobre el sacerdote. El desconocido autor de la copia, Pedro Gutiérrez Pando, dice basarse en el retrato original conservado en la Orden Tercera de San Francisco: «Vera venera/bilis Fratris Joanis, de Bribies/ca, Clerici, Presbyteri que Abulensis / Effigies: ex origina (...) qui apud ter-/tium S. Francisci Hordinem in Se-/(...) item si Abitate permanes (u-/bi ipse met tumulo adque Epitap/hio honorificus iacet) trasumpta / industria simulqu labore Domi-/ni Petri Gutierrez de Pando./ Confecta die IV July anni / autem Domini MDC/LXXXXIII» (Fig. 14) ${ }^{3}$.

El lienzo del Museo es el único que nos habla del pintor Jerónimo López como autor del retrato, pero además, su texto hace hincapié en destacar esta obra como la original — «verdadero, original»- de entre todas las que entonces pudieron existir, de las cuales hoy conocemos tres; esta abundancia de retratos del sacerdote debió ser consecuencia del deseo de poseer la imagen de tan destacado hermano por parte de la entonces floreciente comunidad terciaria.

Según lo expuesto, pensamos que efectivamente la obra conservada en el Museo del Palacio Episcopal de Segovia fue realizada hacia 1619 por el pintor madrileño Jerónimo López Polanco, y por tanto, y a falta de un estudio pormenorizado del ejemplar perteneciente a una colección privada madrileña, este último podría ser considerado como obra contemporánea, bien del mismo autor, o copia fiel de un pintor desconocido.

Pero la abundante iconografía de Briviesca no termina con estos tres lienzos, ya que en la biografía del mismo, que luego comentaremos, volvemos a encontrar la imagen de nuestro ya popular clérigo, esta vez en un grabado realizado por Marcos de Orozco, quien, sin duda, se basó en alguno de los retratos ya comentados ${ }^{4}$.

Respecto a la procedencia de la obra, y pese a la falta de datos a este respecto, lo más probable es que la pintura proceda de la capilla que la Orden Tercera tenía en el convento de San Francisco, lugar donde fue enterrado Briviesca, o incluso de alguna de las sedes que posteriormente tuvieron los terciarios en la ciudad (Fig. 13) ${ }^{5}$.

3 «Verdadero retrato del venerable hermano Juan de Briviesca, clérigo y prebítero abulense: del original que está en la Orden Tercera de San Francisco en Segovia (...) donde permanece (donde él mismo yace honrado con un sepulcro y un epitafio) copiado con la destreza y el trabajo de don Pedro Gutiérrez Pando. Realizado el día 4 de Julio del año del Señor 1693».

${ }^{4}$ Ceán Bermúdez, J. A., Diccionario de los más ilustres profesores de las Bellas Artes en España, Madrid, 1800, tomo III. Gallego, A., Historia del grabado en España, Madrid, 1979, pp. 177-179.

5 La Venerable Orden Tercera, fundada por San Francisco de Asís en 1221, debió establecerse en Segovia al poco tiempo de la fundación del convento en la ciudad a mediados del siglo XIII; alcanzó cierta prosperidad en el siglo XVII ya que entonces construyó su propia capilla adosada a la iglesia del convento de San Francisco, capilla destruida en 1879 con motivo de la instalación en el convento de la Academia de Artillería; posteriormente los terciarios pasaron a la iglesia de San Sebastián, luego a la capilla de la Concepción y capilla de la Paz, frente al obispado, hasta que en 1936 se instalaron definitivamente en el convento franciscano de la calle de la Judería Vieja. Prieto del Pozo, J.: Quinario a San Francisco de Asís, Segovia, 1977, p. 9. 


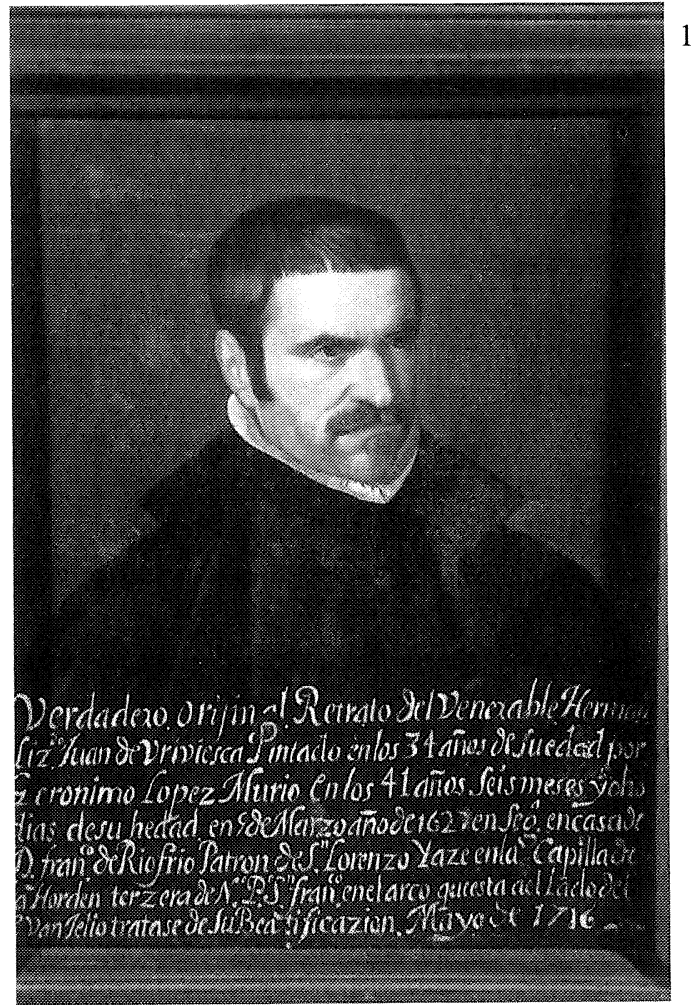

11
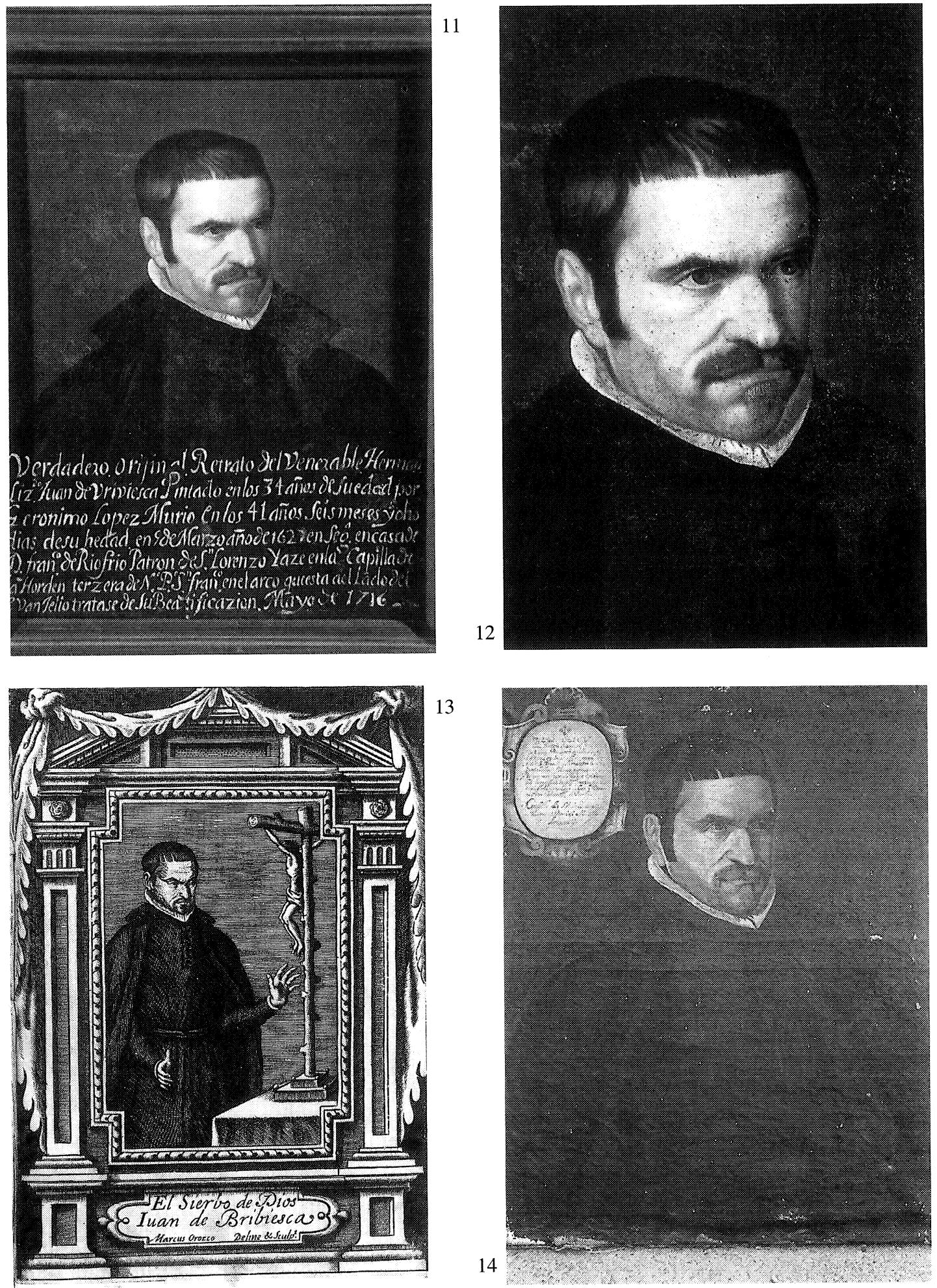

Figs. 11 y 12. Jerónimo López Polanco. Juan de Briviesca, 1619. Conjunto y detalle. Segovia. Museo «Palacio Episcopal».

Fig. 13. Marcos de Orozco. Juan de Briviesca. Vázquez, L.: Vida de Juan de Briviesca. Madrid, 1664. Biblioteca Nacional.

Fig. 14. Pedro Gutiérrez Pando. Juan de Briviesca. Segovia. Colección particular. 


\section{Juan de Briviesca}

Por fortuna existe una biografía de Juan de Briviesca escrita por el sacerdote abulense Luis Vázquez y publicada en Madrid en 1664; Vázquez escribe la obra bajo el patrocinio del Conde de Peñaranda, don Gaspar de Bracamonte y Guzmán, en cuya casa fue criado nuestro clérigo, y dedica la obra a Sebastián de Briviesca, sobrino del venerable sacerdote. El autor afirma que para la relación de su vida se ha basado en los escritos que por mandato de sus confesores hizo el propio Briviesca y también en los datos aportados por «personas fidedignas».

El autor del libro, párroco de San Vicente de Ávila, expone en un marcado estilo «hagiográfico», muy de la época, los hechos más relevantes de la azarosa vida del ejemplar sacerdote, poniendo el acento en los numerosos episodios que describen encuentros místicos, levitaciones, duras penitencias, curaciones milagrosas, trampas y tentaciones del maligno, etc.

Juan de Briviesca nació en Ávila el veintinueve de Agosto de 1585 y recibió el bautismo en la iglesia de San Pedro de esa misma ciudad el día siete de Septiembre; demostró tener desde pequeño un carácter caritativo, virtuoso y sensible hacia el padecimiento de los demás, en especial atención a los pobres; entró como estudiante en la Congregación de nuestra Señora de la Asunción de la Compañía de Jesús, luego pasó a Salamanca para servir a unos hermanos del conde de Peñaranda; su ordenación como sacerdote tuvo lugar en Segovia, aunque su carrera eclesiástica se desarrolló en Ávila, lugar donde entró en contacto con sacerdotes de la Orden Tercera de San Francisco. «En la ciudad de Segovia tenía muchos con quien muy familiarmente comunicaba, eran de santidad y perfección admirable, como se nos dirá en la Crónica que dicen se hace ahora de los siervos y siervas de Dios, que han florecido en virtud y santidad en la Orden Tercera de nuestro Padre San Francisco; tanta virtud y fervor en aquellos varones, eclesiásticos, como seglares conocidos de Juan de Briviesca, que iba a visitarlos a menudo así como ellos venían a visitarle a él» ${ }^{6}$. En efecto, en una de sus frecuentes visitas a la ciudad de Segovia, estando hospedado en casa de don Francisco de Riofrío, terciario franciscano íntimo amigo suyo, cayó gravemente enfermo. En el libro sobre su vida se cuenta con detalle a través del relato de doña Isabel Ramos de la Vega, viuda de don Francisco de Riofrío, la larga agonía de Briviesca, quien padeció durante diecinueve días fortísimos dolores hasta su fallecimiento el cinco de marzo de 1627 a las once de la noche. Dispuso don Francisco de Riofrío que el cuerpo del sacerdote fuese enterrado en la capilla que los Terciarios estaban a punto de concluir en la iglesia del convento de San Francisco de Segovia; para cumplir este deseo, tuvieron que pedir permiso al obispo de la ciudad, don Melchor de Moscoso, quien como prueba de reconocimiento hacia los valores del clérigo, fue él mismo quien bendijo la capilla y ofició la primera misa: «y tomando (el obispo) uno de los zapatos lo llevó consigo estimándolo y venerándolo como a justo y santo» ${ }^{7}$.

El 28 de marzo de 1631 se procede a la apertura de la caja donde fue depositado el cuerpo del clérigo, comprobándose que pese a los cuatro años transcurridos desde el fallecimiento, la conservación del cadáver era admirable y nada indicaba putrefacción: «que teniendo demasiada humedad por la cual debe de oler mal, antes huele bien» y por tanto, los allí presentes, entre los que se encontraba Francisco de Riofrío, atribuyeron la conservación del cuerpo a un hecho de carácter sobrenatural ${ }^{8}$. Pese a no haber localizado la documentación referente al proceso de beatificación, en tramitación en 1716, sin duda este hecho, puesto por escrito ante notario, formó parte de las pruebas presentadas en favor de la beatificación del sacerdote. El proceso, como mu-

\footnotetext{
6 Vàzquez, L., Vida de Juan de Briviesca, Madrid, 1664, p. 152.

7 Vázquez, L., op. cit., p. 152.

8 Vera, J., «El cuerpo del hermano Juan de Briviesca», Estudios Segovianos, XXVII, (1975), p. 171.
} 
chos otros, debió quedar sin resolución favorable a la causa defendida, hemos de suponer, por la Venerable Orden Tercera ${ }^{9}$.

\section{Jerónimo López Polanco}

Nacido en Madrid, posiblemente en el último tercio del siglo XVI, y fallecido en esa misma ciudad en abril de 1626, Jerónimo López Polanco es un artista escasamente conocido en cuanto a su vida y producción artística. Era hermano del también pintor, Andrés López Polanco, digno autor que, sin embargo, muestra en sus obras un estilo tradicional y repetitivo, sin la frescura de las novedades aportadas por el primer naturalismo presente en la obra de Jerónimo ${ }^{10}$. Hasta el momento sólo se habían identificado dos obras de Jerónimo López, dos lienzos con la imagen de la Inmaculada ${ }^{11}$.

La documentación conservada en los archivos madrileños nos permite conocer algunos aspectos interesantes de la vida de Jerónimo López Polanco. Así, el trece de Mayo de 1615, en las capitulaciones matrimoniales previas a su matrimonio con Ana Ruiz, se establecen los compromisos de entrega de dotes por parte de Mateo Vázquez, cuñado de Ana Ruiz, y del propio Jerónimo López; el primero entregará mil cuatrocientos ducados «en ajuar y dineros», mientras que Jerónimo López «a la dicha Ana Ruiz por su honra y virginidad deudos y parientes para más aumento de su dote quinientos ducados los cuales confiesa caben en la décima parte de los bienes que al presente tiene»; se puede deducir por este documento que nuestro pintor disfruta en el momento de su matrimonio de una estable situación económica ${ }^{12}$.

El testamento de Jerónimo López Polanco, otorgado en Madrid con licencia de su madre, María Muñoz, el primero de Abril de 1626, es un documento del mayor interés por la gran información que ofrece ${ }^{13}$; en él, «Jerónimo López Polanco, pintor, vezino y natural desta Villa de Madrid» declara a su mujer, Ana Ruiz, única heredera de todos sus bienes; manda que lo entierren en la Iglesia de San Miguel, junto a su padre «que es sepultura propia»; manda decir un total de quinientas misas; expone que con los catorce mil reales de la dote de su mujer, compró a su hermano Andrés López lo que le correspondía de la herencia de sus padres, y que de dicha herencia no había recibido nada; reconoce deber cincuenta reales a Marcos de Cuevas por unos libros y declara «que tengo enviadas a Indias por orden del señor Cristóbal Gutiérrez Rojo dos partidas de pinturas (...) y por cuenta de las dichas pinturas están en Sevilla cien ducados más o menos. Pido y suplico al dicho señor Cristóbal Gutiérrez Rojo, que siempre me ha hecho merced, procure la cobranza de todo ello. Y quiero que en la cuenta y en todo se esté a lo que

\footnotetext{
${ }^{9}$ El proceso no figura en los índices del Archivo Secreto Vaticano, ni tampoco en las siguientes obras consultadas: Schamoni, W., «Inventarium Procesuum Beatificationis et Canonizationis Bibliothecae Nationalis Parisiensis provenientium ex Archivis S. Rituum Congregationis typis mandatorum inter annos 1662-1809 quod digessit». Hildesheim-Zürich-New York, 1983. Burchi, P., «Catalogus Procesuum Beatificationis et Canonizationis qui in tabulariis et bibliothecis Urbis asservantur». Roma, 1965.

${ }^{10}$ De Andrés López se conservan, entre otros, los retratos de Felipe III del castillo de Nelahozeves o el de Margarita de Austria del museo de Viena, pinturas inmersas en la tradición. Sobre este pintor véase fundamentalmente: Caturla, M. L.: Andrés López Polanco, Cuaderno de Estudios Gallegos, 1956, pp. 389-405 y Urrea, J., «Enrique Trozo y Andrés López, retratistas de obispos», Boletín del Seminario de Arte y Arqueología, Valladolid, 1985, pp. 483-487.

11 Pérez Sánchez, A. E., «Trampantojos a lo divino», Lecturas de Arte, III (1992), pp. 47-148. Son dos Inmaculadas basadas en una escultura de Gregorio Fernández; una se conserva en Canarias, la otra en Dueñas, Palencia; ambas están firmadas, la primera fechada en 1629, fecha que no debe corresponder al año de la realización de la obra, pues el autor falleció en 1626.

12 (1615-V-13, Madrid) Archivo Histórico de Protocolos. Protocolo 2560, fóls.1148-1150. Capitulaciones matrimoniales de Jerónimo López y Ana Ruiz.

13 Tanto el testamento como el acta del libro de enterramientos los recoge Agulló y Cobo, M. en: Noticias sobre pintores madrileños de los siglos XVI y XVII, Granada, 1978, pp. 88-89.
} 
este dicho Cristóbal Gutiérrez Rojo dijere en razón de ello» ${ }^{14}$. Especial interés tiene este párrafo, pues ilustra acerca de lo que en ese período supuso para muchos pintores el mercado americano como salida comercial para su trabajo, ante el competitivo mercado artístico de la corte. El tono de la frase y la confianza que nuestro pintor deposita en el intermediario o marchante, prueba la existencia de una fluida y larga relación comercial entre ambos ${ }^{15}$.

El último documento en relación con Jerónimo López es la anotación consignada el catorce de abril de 1626 en el libro de enterramientos de San Miguel, libro conservado en la parroquia de San Justo de Madrid: «En la calle de la Compañía (...) murió Gerónimo López, pintor (...) mandó enterrarse en su sepultura en San Miguel (...) mandó cien misas de alma y cuatrocientas ordinarias. Testamentarios Andrés López, pintor (...) y Cristóbal Ruiz, mercader de lienzos» ${ }^{16}$.

Todo lo aportado nos ofrece el perfil, aún incompleto, de un notable pintor madrileño del primer tercio del siglo XVII cuyo catálogo se compone, hasta el momento, por tres obras seguras y una cuarta con muchas posibilidades de ser incluida en el mismo, el retrato de Juan de Briviesca conservado en Madrid.

Comparando dos de estas obras, el retrato de Briviesca y la Inmaculada de Dueñas, descubrimos en esta última ciertas novedades respecto a la primera, novedades que se explican tanto por la diferencia en cuanto a tema o finalidad de las mismas, como por percibirse cierta evolución en la trayectoria del autor. Así, en cuanto al color, aunque siempre comedido, sustituye la austeridad dominante en el retrato del Museo del Palacio Episcopal por el sutil cromatismo que envuelve la composición de la Inmaculada de la Iglesia de la Asunción de Dueñas. Esta obra, interesante en su conjunto, alcanza altas cotas de calidad en algunos fragmentos especialmente bellos, como el grupo de cabezas de querubines situadas a los pies de la Virgen, las flores de la parte baja de la composición, o el mismo rostro de la Virgen, todos ellos tratados con un sobrio y elegante estilo naturalista ya presente en el retrato del clérigo Juan de Briviesca.

Pintor con la suficiente calidad como para haber conseguido destacar en el ambiente artístico madrileño del primer tercio del siglo XVII, su figura se nos muestra hoy algo más definida, aunque aún sin la claridad con la que sus obras reflejan su maestría.

CARLOS SÁNCHEZ DíEZ

Museo «Palacio Episcopal», Segovia

\section{JUAN DE BORGOÑA, PEDRO CISNEROS Y CRISTÓBAL DE VILLARREAL EN GALAPAGAR (MADRID)}

Desde que don Diego Angulo Íñiguez publicase hace poco más de cuarenta años su valiosa monografía sobre Juan de Borgoña, no se ha vuelto a afrontar una visión global de esta personalidad clave para la pintura del renacimiento castellano ${ }^{1}$. Quedan todavía por clarificar, como

\footnotetext{
14 (1626-IV-1, Madrid) Archivo Histórico de Protocolos. Protocolo número 5874, fóls. 9-11. Testamento de Jerónimo López Polanco.

${ }^{15}$ No es de extrañar la presencia de una obra de Jerónimo López Polanco en Canarias, escala del viaje a América.

${ }^{16}$ Agulló y Cobo, M., op. cit., p. 89.

1 D. Angulo Íñiguez, Juan de Borgoña, Madrid, 1954. Antereior a la obra de Angulo, C. R. Post, «The beginning of the Renaissance in Castille and Leon», en A history of spanish painting, vol. IX, I, Cambridge, Massachusetts, 1947, pp. 162-234, donde se incluye la bibliografía anterior.
} 